\title{
THE SCHUR-HORN THEOREM FOR OPERATORS WITH FINITE SPECTRUM
}

\author{
B. V. RAJARAMA BHAT AND MOHAN RAVICHANDRAN
}

(Communicated by Marius Junge)

\begin{abstract}
The carpenter problem in the context of $I I_{1}$ factors, formulated by Kadison, asks: Let $\mathcal{A} \subset \mathcal{M}$ be a masa in a type $I I_{1}$ factor and let $E$ be the normal conditional expectation from $\mathcal{M}$ onto $\mathcal{A}$. Then, is it true that for every positive contraction $A$ in $\mathcal{A}$, there is a projection $P$ in $\mathcal{M}$ such that $E(P)=A$ ? In this note, we show that this is true if $A$ has finite spectrum. We will then use this result to prove an exact Schur-Horn theorem for positive operators with finite spectrum in type $I I_{1}$ factors and an approximate Schur-Horn theorem for general positive operators in type $I I_{1}$ factors.
\end{abstract}

\section{INTRODUCTION}

Let $\mathcal{A}$ be a masa in a $I I_{1}$ factor $\mathcal{M}$ and $E$ the normal conditional expectation from $\mathcal{M}$ to $\mathcal{A}$. Kadison, in [7, asked the following question:

Question 1.1 (Kadison's carpenter problem). Given any positive contraction $B$ in $\mathcal{A}$, does there exist a projection $P$ in $\mathcal{M}$ so that $E(P)=B$ ?

We refer the reader to the above cited paper for the discussion leading up to this problem. The best result to date is the result of [5] that says the following:

Theorem 1.2 (Dykema, Fang, Hadwin, Smith). Kadison's carpenter problem has an affirmative solution for generator masas in $L\left(F_{2}\right)$. Also, for any positive contraction $B$ in a Cartan masa $\mathcal{A}$ in the hyperfinite $I I_{1}$ factor $\mathcal{R}$, there is an automorphism $\theta$ of $\mathcal{A}$ and a projection $P$ in $\mathcal{R}$ so that $E_{\mathcal{A}}(P)=\theta(B)$.

There are several consequences of this result that the reader can work out independently. For general $I I_{1}$ factors, far less is known. Indeed, everything that is known so far with the exception of the result mentioned above and some extensions proved in the same paper, is a straightforward interpretation of results for matrices. For instance, the matricial Schur-Horn theorem guarantees that $\lambda I$ can be lifted if $\lambda$ is a rational number, but it is not known if irrational multiples of the identity can be lifted to projections. In this note we show that this is indeed the case. It will follow that elements with finite spectrum can be lifted to projections.

In this note, we will work in a slightly more general context. Kadison's carpenter problem is a special case of a majorization problem for von Neumann algebras. The notion of majorization in von Neumann algebras goes back at least to Hiai's work [6] in the 1980s. The following definition is an extension of classical majorization to the continuous case.

Received by the editors February 9, 2012 and, in revised form, September 4, 2012.

2010 Mathematics Subject Classification. Primary 46L10; Secondary 46L54. 
Definition 1.3 (Majorization). Given two self-adjoint operators $A, S$ in a finite factor $(\mathcal{M}, \tau)$, say that $A$ is majorized by $S$ and denoted by $A \prec S$ if

$$
\tau(f(A)) \leq \tau(f(S))
$$

for every continuous convex real valued function $f$ defined on a closed interval $[c, d]$ containing the spectra of both $A$ and $S$.

The condition implies that $\tau(A)=\tau(S)$. Majorization can be expressed in several ways, and these equivalences can be found in [6] and the references therein. A natural extension of Kadison's problem was formulated by Kadison and Arveson in [4].

Question 1.4 (Arveson and Kadison's Schur Horn problem). Let $A$ be a positive element in $\mathcal{A}$ and $S$ a positive element in $\mathcal{M}$ such that $A \prec S$. Then, is it true that there exists an element $T$ in $\left.\mathcal{O}(S)=\overline{\left\{U S U^{*}, U \in \mathcal{U}(M)\right.}\right\}$ " such that $E(T)=A$ ?

One does need to take the norm closure; see the example following Lemma 5.5 in the same paper.

This problem was solved in the affirmative for the generator and radial masas in the free group factors in [5], where it was also solved modulo an automorphism of the masa for Cartain masas in the hyperfinite $I I_{1}$ factor. In this note, we will work with general masas inside general type $I I_{1}$ factors. Our main result is the following theorem whose proof is an adaptation of the best known proof of the matricial Schur Horn theorem.

Theorem 1.5. Let $\mathcal{A}$ be a masa in a $I I_{1}$ factor $\mathcal{M}$ and let $E$ be the normal conditional expectation from $\mathcal{M}$ to $\mathcal{A}$. Let $A \in \mathcal{A}$ and $S \in \mathcal{M}$ be positive operators with finite spectrum such that $A \prec S$. Then, there is a unitary $U$ in $\mathcal{M}$ so that $E\left(U S U^{*}\right)=A$.

The theorem says that the Schur-Horn problem can be solved when both elements have finite spectrum. Routine calculations will then allow us to adapt the above theorem to deduce an approximate Schur-Horn theorem for general operators in a $I I_{1}$ factor.

Theorem 1.6. Let $S$ be a positive operator in $\mathcal{M}$. Then, the norm closure of $E(\mathcal{U}(S))$ equals $\left\{A \in \mathcal{A}^{+} \mid A \prec S\right\}$.

In particular, letting $\mathcal{O}(S)={\overline{\left\{U S U^{*} \mid U \in \mathcal{U}(\mathcal{M})\right.}}^{\|}$, we have that

$$
\overline{E(\mathcal{O}(S))} \|^{\|}=\{A \in \mathcal{A} \mid A \prec S\} .
$$

The conjectured Schur-Horn theorem of Arveson and Kadison says that we do not need to take the norm closure for equality, something that we are unable to prove in this paper. A weaker version of our theorem where the $\sigma-\mathrm{SOT}$ closure was taken in the place of the norm closure was proved by Argerami and Massey in [3. Also, the above result was established for Cartan masas in the hyperfinite $I I_{1}$ factors (and thus for general semi-regular masas; see [11]) in [5].

The paper has four sections apart from the introduction. In section 2 , we show that scalars can be lifted to projections. In section 3, we push this through to show that the Schur-Horn problem can be solved for operators with finite spectrum. Section 4 contains the approximate Schur-Horn theorem. There is then a last section consisting of some remarks and observations. 
Some words on notation: Given two operators $A, B$ inside a von Neumann algebra $\mathcal{M}$ such that there is a projection $P$ inside $\mathcal{M}$ with $A=P A P$ and $B=(I-P) B(I-P)$, in order to stress the fact that $A$ and $B$ live under the auspices of orthogonal projections, we will use the expression $A \oplus B$ to denote their sum. Next, given a self-adjoint operator $A$ and a Borel measurable subset $X$ of the real line, the expression $E_{A}(X)$ will denote the spectral projection of $A$ corresponding to the subset $X$. This notation conflicts with the usage of $E_{\mathcal{A}}(A)$ or simply $E(A)$ where $\mathcal{A}$ is a subalgebra of $\mathcal{M}$, which denotes the image under a conditional expectation $E$; hopefully this will not cause any confusion.

\section{LIFTING SCALARS}

The carpenter theorem for matrices mentioned in [7] ensures that rational multiples of the identity can be lifted to projections. In this section, we will show that irrational multiples of the identity can be lifted to as well. We begin with a simple observation.

Lemma 2.1. Let $P$ be a projection in a masa $\mathcal{A}$ inside a type $I I_{1}$ factor $\mathcal{M}$ and let $\lambda, a, b$ be positive scalars such that $\tau(S)=\lambda$, where $S=a P+b(I-P)$. Then, there is a unitary $U$ in $\mathcal{M}$ and a projection $Q$ in $\mathcal{A}$ such that letting $T=U S U^{*}$, we have that

(1) $E(Q T Q)=\lambda Q$.

(2) $(I-Q) T(I-Q)=c R+d(I-Q-R)$ for some projection $R$ in $\mathcal{A}$ with $R \leq I-Q$ and positive numbers $c, d$.

(3) $\tau(Q) \geq \frac{1}{3}$.

Proof. The lemma is trivial if $a=b$, for then $a=b=\lambda$ and there is nothing to prove. We assume without loss of generality that $a>b$. Since $\tau(S)=\lambda$, we must then have that $a>\lambda>b$. We may also assume that $\tau(P) \leq \frac{1}{2}$. For, suppose we have proved the lemma in this case; the result when $\tau(P)>\frac{1}{2}$ can be derived by applying the lemma to $I-S$ and $(1-\lambda) I$. We therefore assume that $\tau(P) \leq \frac{1}{2}$.

Let $k$ be the largest integer such that $(k+1) \tau(P) \leq 1$. Since $\tau(P) \leq \frac{1}{2}$, the positive integer $k$ must be at least 1 . Pick projections $Q_{1}, \cdots, Q_{k}$, each of trace $\tau(P)$ in $\mathcal{A}$ that are mutually orthogonal and also orthogonal to $P$. Let $V_{1}, \cdots, V_{k}$ be partial isometries in $\mathcal{M}$ such that

(1) $V_{1}^{*} V_{1}=Q_{1}$ and $V_{1} V_{1}^{*}=P$.

(2) For $2 \leq i \leq k, V_{i} V_{i}^{*}=Q_{i-1}$ and $V_{i}^{*} V_{i}=Q_{i}$.

Pick $\theta_{1}$ such that $a \cos ^{2}\left(\theta_{1}\right)+b \sin ^{2}\left(\theta_{1}\right)=\lambda$ and let $U_{1}$ be the operator

$$
U_{1}=\cos \left(\theta_{1}\right) P+\sin \left(\theta_{1}\right) V_{1}-\sin \left(\theta_{1}\right) V_{1}^{*}+\cos \left(\theta_{1}\right) Q_{1}+\left(I-P-Q_{1}\right) .
$$

We will identify the above operator with the operator matrix (using $V_{1}$ as the matrix unit $E_{12}$ ), an identification that is standard:

$$
U_{1}=\left(\begin{array}{ccc}
\cos \left(\theta_{1}\right) & \sin \left(\theta_{1}\right) & 0 \\
-\sin \left(\theta_{1}\right) & \cos \left(\theta_{1}\right) & 0 \\
0 & 0 & I
\end{array}\right) .
$$


Under this same identification, $S$ is the operator

$$
S=\left(\begin{array}{lll}
a & 0 & 0 \\
0 & b & 0 \\
0 & 0 & b
\end{array}\right)
$$

Let $S_{1}=U_{1} S U_{1}^{*}$. It is easy to check that $U_{1}$ is a unitary and that

$$
S_{1}=\left(\begin{array}{ccc}
a \cos ^{2}\left(\theta_{1}\right)+b \sin ^{2}\left(\theta_{1}\right) & * & 0 \\
* & a \sin ^{2}\left(\theta_{1}\right)+b \cos ^{2}\left(\theta_{1}\right) & 0 \\
0 & 0 & b
\end{array}\right)=\left(\begin{array}{ccc}
\lambda & * & 0 \\
* & a_{1} & 0 \\
0 & 0 & b_{1}
\end{array}\right) \text {, }
$$

where $a_{1}=a \sin ^{2}\left(\theta_{1}\right)+b \cos ^{2}\left(\theta_{1}\right)$ and $b_{1}=b$. Equating traces,

$$
\lambda \tau(P)+a_{1} \tau(P)+b_{1}(1-2 \tau(P))=\lambda .
$$

Since $b_{1}=b<\lambda$, we must have that $a_{1}>\lambda$ and a fortiori $a_{1}>b_{1}$.

Now, continue as above. Pick $\theta_{2}$ such that $a_{1} \cos ^{2}\left(\theta_{2}\right)+b_{1} \sin ^{2}\left(\theta_{2}\right)=\lambda$ and let $U_{2}$ be the operator

$$
U_{2}=\cos \left(\theta_{2}\right) Q_{1}+\sin \left(\theta_{2}\right) V_{2}-\sin \left(\theta_{2}\right) V_{2}^{*}+\cos \left(\theta_{2}\right) Q_{2}+\left(I-Q_{1}-Q_{2}\right) .
$$

We may write the unitary $U_{2}$ as

$$
U_{2}=\left(\begin{array}{cccc}
I & 0 & 0 & 0 \\
0 & \cos \left(\theta_{2}\right) & \sin \left(\theta_{2}\right) & 0 \\
0 & -\sin \left(\theta_{2}\right) & \cos \left(\theta_{2}\right) & 0 \\
0 & 0 & 0 & I
\end{array}\right)
$$

and let $S_{2}=U_{2} S_{1} U_{2}^{*}$. We have that

$$
S_{2}=\left(\begin{array}{cccc}
\lambda & * & * & 0 \\
* & \lambda & * & 0 \\
* & * & a_{2} & 0 \\
0 & 0 & 0 & b_{2}
\end{array}\right)
$$

where $a_{2}=a_{1} \sin ^{2}\left(\theta_{2}\right)+b_{1} \cos ^{2}\left(\theta_{2}\right)$ and $b_{2}=b_{1}$. Equating traces,

$$
2 \lambda \tau(P)+a_{2} \tau(P)+b_{2}(1-3 \tau(P))=\lambda .
$$

Since $b_{2}=b_{1}=b<\lambda$, we must have that $a_{2}>\lambda$ and a fortiori $a_{2}>b_{2}$.

Proceeding this way $k-2$ more times, we get an operator $S_{k}$ of the form

$$
S_{k}=\left(\begin{array}{cccccc}
\lambda & * & \ldots & * & * & 0 \\
* & \lambda & \ldots & * & * & 0 \\
\vdots & \vdots & \ddots & \vdots & * & 0 \\
* & * & * & \lambda & * & 0 \\
* & * & * & * & a_{k} & 0 \\
0 & 0 & 0 & 0 & 0 & b_{k}
\end{array}\right)
$$

Let $Q=P+Q_{1}+\cdots+Q_{k-1}$ (if $k=1$, let $\left.Q=P\right)$. We see that

(1) $E\left(Q S_{k} Q\right)=\lambda Q$. This is because

$$
\begin{aligned}
E\left(Q S_{k} Q\right) & =E\left(P S_{k} P\right)+E\left(Q_{1} S_{k} Q_{1}\right)+\cdots+E\left(Q_{k-1} S_{k} Q_{k-1}\right) \\
& =\lambda P+\lambda Q_{1}+\cdots+\lambda Q_{k-1} \\
& =\lambda Q .
\end{aligned}
$$

( $S_{k}$ is the operator $T$ promised in the statement of the lemma). 
(2) $(I-Q) S_{k}(I-Q)$ has a two point spectrum in $(I-Q) M(I-Q)$.

(3) $\tau(Q)=k \tau(P)$. Since $(k+1) \tau(P) \leq 1<(k+2) \tau(P)$, we see that

$$
\tau(Q)=k \tau(P)=\frac{k}{k+2}(k+2) \tau(P)>\frac{k}{k+2} \geq \frac{1}{3} .
$$

The lemma follows.

We now use a simple limit argument to show that scalar multiples of the identity can be lifted to projections.

Theorem 2.2. Let $\mathcal{A}$ be a masa in a $I I_{1}$ factor $\mathcal{M}$ and let $E$ be the normal conditional expectation from $\mathcal{M}$ to $\mathcal{A}$. Then for any $0 \leq \lambda \leq 1$, there is a projection $P$ in $\mathcal{M}$ such that $E(P)=\lambda I$.

Proof. Let $P_{0}$ be any projection of trace $\lambda$ in $\mathcal{A}$. Using Lemma 2.1, construct a unitary $U_{1}$ and a projection $Q_{1}$ in $\mathcal{A}$ such that, letting $P_{1}=U_{1} P_{0} U_{1}^{*}$,

(1) $\tau\left(Q_{1}\right) \geq \frac{1}{3}$

(2) $E\left(Q_{1} P_{1} Q_{1}\right)=\lambda Q_{1}$.

(3) $\left(I-Q_{1}\right) P_{1}\left(I-Q_{1}\right)$ has two point spectrum in $\left(I-Q_{1}\right) M\left(I-Q_{1}\right)$.

Let $R_{1}=Q_{1}$. Next, for $k=2,3, \cdots$, apply Lemma 2.1 to $\lambda\left(I-R_{k-1}\right)$ and $\left(I-R_{k-1}\right) Q_{k-1}\left(I-R_{k-1}\right)$ inside the $I I_{1}$ factor $\left(I-R_{k-1}\right) M\left(I-R_{k-1}\right)$ to construct a unitary $U_{k}$ and a projection $Q_{k}$ in $\left(I-R_{k-1}\right) M\left(I-R_{k-1}\right)$ and let

$$
R_{k}=Q_{1} \oplus Q_{2} \oplus \cdots \oplus Q_{k} \quad \text { and } \quad P_{k}=\left(R_{k-1} \oplus U_{k}\right) P_{k-1}\left(R_{k-1} \oplus U_{k}\right)^{*} .
$$

Here we identify $Q_{k}$, which is a projection in $\left(I-R_{k-1}\right) M\left(I-R_{k-1}\right)$, with a projection in $\mathcal{M}$ dominated by $I-R_{k-1}$. Note that $P_{k}$ is a projection. We have that

(1) $E\left(Q_{k} P_{k} Q_{k}\right)=\lambda Q_{k}$, and thus

$$
E\left(R_{k} P_{k} R_{k}\right)=\sum_{m=1}^{k} E\left(Q_{m} P_{m} Q_{m}\right)=\sum_{m=1}^{k} \lambda Q_{m}=\lambda R_{k}
$$

(2) $\tau\left(I-R_{k}\right) \leq \frac{2}{3} \tau\left(I-R_{k-1}\right) \leq\left(\frac{2}{3}\right)^{k}$, and hence $R_{k}$ converges to $I$ strongly.

(3) $\left(I-R_{k}\right) P_{k}\left(I-R_{k}\right)$ has two point spectrum in $\left(I-R_{k}\right) \mathcal{M}\left(I-R_{k}\right)$.

(4) We also have that $R_{k-1} P_{k-1} R_{k-1}=R_{k-1} P_{k} R_{k-1}$, and thus

$$
R_{l}\left(P_{m}-P_{n}\right) R_{l}=0 \quad \text { for any } n, m \geq l .
$$

We now claim that $P_{k}$ converges in the strong operator topology to a projection that we will call $P$ and also that $E(P)=\lambda I$. For the first claim, since the projections $R_{k}$ converge strongly to $I$ by statement (2) above, for any $\epsilon>0$ there is an $N$ so that $\left\|\left(I-R_{N}\right)\right\|_{2}<\epsilon$. For $n, m \geq N$,

$$
\left\|\left(P_{n}-P_{m}\right)\right\|_{2} \leq\left\|R_{N}\left(P_{n}-P_{m}\right) R_{N}\right\|_{2}+2\left\|\left(I-R_{N}\right)\left(P_{n}-P_{m}\right)\right\|_{2} .
$$

The first term is zero by (2.1). For the second term,

$$
\left\|\left(I-R_{N}\right)\left(P_{n}-P_{m}\right)\right\|_{2} \leq\left\|I-R_{N}\right\|_{2}\left\|P_{n}-P_{m}\right\| \leq 2 \epsilon .
$$


Thus, $\left\|\left(P_{n}-P_{m}\right)\right\|_{2} \leq 4 \epsilon$ and the sequence $\left\{P_{n}\right\}$ is strongly convergent. Let $P$ be the limit projection. For the second claim,

$$
\begin{aligned}
\|E(P)-\lambda I\|_{2} & =\lim \left\|E\left(P_{n}\right)-\lambda I\right\|_{2} \\
& =\lim \left\|\lambda R_{n}+E\left(\left(I-R_{n}\right) P_{n}\left(I-R_{n}\right)\right)-\lambda I\right\|_{2} \\
& =\lim \left\|-\lambda\left(I-R_{n}\right)+E\left(\left(I-R_{n}\right) P_{n}\left(I-R_{n}\right)\right)\right\|_{2} \\
& \leq \lim \lambda\left\|\left(I-R_{n}\right)\right\|_{2}+\left\|\left(I-R_{n}\right) P_{n}\left(I-R_{n}\right)\right\|_{2} \\
& \leq \lim \lambda\left(\frac{2}{3}\right)^{n}+\left\|P_{n}\right\|\left(\frac{2}{3}\right)^{n} \\
& \leq \lim (\lambda+1)\left(\frac{2}{3}\right)^{n} \\
& =0 .
\end{aligned}
$$

We conclude that $E(P)=\lambda I$.

We record a simple proposition.

Proposition 2.3. Let $A$ be a positive contraction in $\mathcal{A}$ that can be written as $A=\sum_{n} \lambda_{n} E_{n}$, where the $E_{n}$ 's are orthogonal projections summing up to $I$. Then, there is a projection $P$ in $\mathcal{M}$ such that $E(P)=A$.

Proof. The element $A$ may be written as $A=\sum_{n=1}^{\infty} \lambda_{n} E_{n}$, where the $E_{n}$ 's are mutually orthogonal projections in $\mathcal{A}$ summing up to 1 and $0 \leq \lambda_{n} \leq 1$ for every $n$. The algebra $E_{n} \mathcal{M} E_{n}$ is a type $I I_{1}$ factor, and we may find a projection $P_{n}$ in $E_{n} M E_{n}$ such that $E_{A E_{n}}\left(P_{n}\right)=\lambda_{n} E_{n}$ for every $n$. Let $P$ be the projection $\sum_{n=1}^{\infty} P_{n}$. Here, we are identifying $P_{n}$, which is a projection in $E_{n} M E_{n}$, with a projection in $\mathcal{M}$ that is dominated by $E_{n}$. Then,

$$
E(P)=\sum_{n=1}^{\infty} E\left(P_{n}\right)=\sum_{n=1}^{\infty} E\left(E_{n} P_{n} E_{n}\right)=\sum_{n=1}^{\infty} \lambda_{n} E_{n}=A .
$$

\section{The SCHUR-HORN THEOREM FOR OPERATORS WITH FINITE SPECTRUM}

We will now bootstrap the theorem in the previous section to get a Schur-Horn theorem for positive operators with finite spectrum. Recall the following reformulation of majorization in $I I_{1}$ factors. Let $A, S$ be positive contractions in a type $I I_{1}$ factor $\mathcal{M}$ and let $f, g:[0,1] \rightarrow[0,1]$ be the (essentially unique, right-continuous, non-increasing) functions (the $s$ number functions), see for instance $[8$, which satisfy

$$
\tau\left(A^{n}\right)=\int_{0}^{1} f^{n}(r) d m(r) \quad \text { and } \quad \tau\left(S^{n}\right)=\int_{0}^{1} g^{n}(r) d m(r) \quad \text { for } n=0,1, \cdots .
$$

Here $m$ stands for Lebesgue measure on $\mathbb{R}$. Then $A \prec S$ iff (see [6]),

$$
\int_{0}^{t} f(r) d m(r) \leq \int_{0}^{t} g(r) d m(r), 0 \leq t \leq 1, \quad \text { and } \quad \int_{0}^{1} f(r) d m(r)=\int_{0}^{1} g(r) d m(r) .
$$

Lemma 3.1. Let $A=\lambda_{1} E_{1} \oplus \lambda_{2} E_{2}$, where $E_{1}+E_{2}=I$ and $\lambda_{1} \geq \lambda_{2} \geq 0$, and $S=\mu_{1} F_{1} \oplus \mu_{2} F_{2}$, where $F_{1}+F_{2}=I$ and $\mu_{1}>\mu_{2} \geq 0$, be two operators in a $I I_{1}$ factor with $\tau(A)=\tau(S)$. If $\mu_{1} \geq \lambda_{1}$ and $\mu_{2} \leq \lambda_{2}$, then $A \prec S$. 
Proof. It is easy to see that if $B$ is a positive contraction, then $B \prec P$ for any projection $P$ with $\tau(P)=\tau(B)$. Let $c=\frac{1}{\mu_{1}-\mu_{2}}$ and $d=-\frac{\mu_{2}}{\mu_{1}-\mu_{2}}$. The operator $c S+d I$ may be checked to equal $F_{1}$. It is hence a projection, and of course $\tau(c S+d I)=\tau(c A+d I)$.

$$
c A+d I=\left(c \lambda_{1}+d\right) E_{1}+\left(c \lambda_{2}+d\right) E_{2}=\frac{\lambda_{1}-\mu_{2}}{\mu_{1}-\mu_{2}} E_{1}+\frac{\lambda_{2}-\mu_{2}}{\mu_{1}-\mu_{2}} E_{2} .
$$

Since $\lambda_{2} \leq \lambda_{1}, \lambda_{2} \geq \mu_{2}$ and $\lambda_{1} \leq \mu_{1}$, we have that

$$
0 \leq \frac{\lambda_{2}-\mu_{2}}{\mu_{1}-\mu_{2}} \leq \frac{\lambda_{1}-\mu_{2}}{\mu_{1}-\mu_{2}} \leq \frac{\mu_{1}-\mu_{2}}{\mu_{1}-\mu_{2}}=1 .
$$

Thus, $c A+d I$ is a positive contraction. By the observation in the first line of the proof, $c A+d I \prec c S+d I$ and therefore $A \prec S$.

Lemma 3.2. Let $A=\lambda_{1} E_{1} \oplus \lambda_{2} E_{2}$ and $S=\mu_{1} E_{1} \oplus \mu_{2} E_{2}$, where $E_{1}+E_{2}=I$, be positive operators in a type II $I_{1}$ factor $\mathcal{M}$ with the same trace. If $\lambda_{1} \leq \mu_{1}$, then $A \prec S$.

Proof. It is easy to see that we must have $\mu_{2}<\lambda_{2}$. The lemma now follows from Lemma 3.1

Lemma 3.3. Let $A$ be a self-adjoint operator and $S$ a positive contraction in a $I I_{1}$ factor so that $A \prec S$. Then $A$ is a positive contraction as well.

Proof. Routine verification.

Proposition 3.4. Let $S$ be a positive operator in $\mathcal{M}$ with two point spectrum and let $A$ be a positive contraction in $\mathcal{A}$ that has finite spectrum and so that $A \prec S$. Then, there is a unitary $U$ in $\mathcal{M}$ such that $E\left(U S U^{*}\right)=A$.

Proof. Write $S=\mu_{1} F_{1} \oplus \mu_{2} F_{2}$, where $\mu_{1} \geq \mu_{2}$ and $F_{1} \oplus F_{2}=I$. Let $c=\frac{1}{\mu_{1}-\mu_{2}}$ (note that $c>0$ ) and $d=-\frac{\mu_{2}}{\mu_{1}-\mu_{2}}$. The operator $c S+d I$ may be checked to equal $F_{1}$ and is hence a projection. We also have that $c A+d I \prec c S+d I=F_{1}$. By Lemma 3.3. $c A+d I$ must actually be a positive contraction. Also, of course, $\tau(c S+d I)=\tau(c A+d I)$. Now, by Proposition 2.3, there is a unitary $U$ so that $E\left(U(c S+d I) U^{*}\right)=c A+d I$, and hence $E\left(U S U^{*}\right)=A$.

When one or both operators have finite spectrum, majorization reduces to a simple condition.

Lemma 3.5. Let $A, S$ be positive operators in a $I I_{1}$ factor with $\tau(A)=\tau(S)$ and let $f, g$ be the $s$-number functions of $A, S$ respectively, as above. Suppose $A$ has finite spectrum, i.e., the $s$-number function $f$ for $A$ has the form

$$
f=\sum_{n=1}^{N} \lambda_{n} \chi_{\left[s_{n-1}, s_{n}\right)}
$$

for some natural number $N$ and some sequences $0=s_{0}<s_{1}<\cdots<s_{N}=1$ and $0 \leq \lambda_{N}<\lambda_{N-1}<\cdots<\lambda_{1}$. Then, $A \prec S$ iff for $n=1,2, \cdots, N$,

$\int_{0}^{s_{n}} f(r) d m(r) \leq \int_{0}^{s_{n}} g(r) d m(r)$ or, equivalently, $\tau\left(A E_{A}\left(\left[0, s_{n}\right)\right)\right) \leq \tau\left(S E_{S}\left(\left[0, s_{n}\right)\right)\right)$. 
Proof. Routine verification.

We now prove the promised special case of the Schur-Horn theorem.

Theorem 3.6 (The Schur-Horn theorem for operators with finite spectrum in a $I I_{1}$ factor). Let $A$ and $S$ be positive operators with finite spectrum in $\mathcal{A}$ and $\mathcal{M}$ respectively and so that $A \prec S$. Then, there is a unitary $U$ in $\mathcal{M}$ so that $E\left(U S U^{*}\right)=A$.

Proof. We assume that $A$ and $S$ have spectrum consisting of $N$ and $M$ points respectively. Write $A=\sum_{n=1}^{N} \lambda_{n} E_{n}$ and $S=\sum_{n=1}^{M} \mu_{n} F_{n}$, where the $\left\{\lambda_{n}\right\}_{1}^{N}$ (respectively, the $\left\{\mu_{n}\right\}_{1}^{M}$ ) are distinct. Assume now that the theorem has been proved whenever the operators have finite spectra and the cumulative number of eigenvalues is less than $N+M$.

We may assume that none of the $\lambda_{i}$ equal any of the $\mu_{j}$. For suppose $\lambda_{i}=\mu_{j}$. Assume that $\tau\left(E_{i}\right) \leq \tau\left(F_{j}\right)$; the other case is handled similarly. We may, after conjugating by a unitary, write $A=\lambda_{i} E_{i} \oplus\left(A-\lambda_{i} E_{i}\right)$ and $S=\mu_{j} E_{i} \oplus\left(S-\mu_{j} E_{i}\right)=$ $\lambda_{i} E_{i} \oplus\left(S-\lambda_{i} E_{i}\right)$. Clearly, $A-\lambda_{i} E_{i} \prec S-\lambda_{i} E_{i}$, and it is enough to prove the theorem for $A-\lambda_{i} E_{i}$, which has at most $N-1$ point spectrum in $\left(I-E_{i}\right) \mathcal{A}$, and $S-\lambda_{i} E_{i}$, which has at most $M$ point spectrum inside $\left(I-E_{i}\right) \mathcal{M}\left(I-E_{i}\right)$. We therefore assume that none of the $\lambda_{i}$ equal any of the $\mu_{j}$.

Since $\mathcal{A}$ is isomorphic to $L^{\infty}([0,1], d m)$, we may find a maximal nest of projections $\left\{P_{t}: 0 \leq t \leq 1\right\}$ in $\mathcal{A}$ with $P_{t} \leq P_{s}$ for $0 \leq t \leq s \leq 1$ and $\tau\left(P_{t}\right)=t$ for $0 \leq t \leq 1$. Since $A$ (respectively $S$ ) has $N$ (respectively $M$ ) point spectrum, we may, after conjugating $A$ and $S$ by unitaries, assume that $A$ and $S$ have the form

$$
A=\sum_{n=1}^{N} \lambda_{n}\left(P_{s_{n}}-P_{s_{n-1}}\right) \quad \text { and } \quad S=\sum_{n=1}^{M} \mu_{n}\left(P_{t_{n}}-P_{t_{n-1}}\right)
$$

for sequences $0=s_{0}<s_{1}<s_{2}<\cdots<s_{N}=1$ and $0=t_{0}<t_{1}<t_{2}<\cdots<t_{M}=1$ and positive scalars $\lambda_{1}>\lambda_{2}>\cdots>\lambda_{N} \geq 0$ and $\mu_{1}>\mu_{2}>\cdots>\mu_{M} \geq 0$.

Reindex the set $\left\{s_{1}, \cdots, s_{N-1}\right\} \cup\left\{t_{1}, \cdots, t_{M-1}\right\}$ by $\left\{r_{1}, \cdots, r_{L}\right\}$, where $r_{1}<$ $r_{2}<\cdots<r_{L-1}$, and let $r_{L}=1$. Then, we may write

$$
A=\sum_{n=1}^{L} \gamma_{n}\left(P_{r_{n}}-P_{r_{n-1}}\right) \quad \text { and } \quad f=\sum_{n=1}^{L} \delta_{n}\left(P_{r_{n}}-P_{r_{n-1}}\right) \text {, }
$$

where $\gamma_{n}=\lambda_{m}$ for the unique value $m$ so that $\left[r_{n-1}, r_{n}\right) \subset\left[s_{m-1}, s_{m}\right)$ and similarly for the numbers $\delta_{n}$.

We will prove the theorem by induction on $L$. When $L=1, A$ and $S$ are scalars, and thus $A=S=\tau(A) I$ and the theorem is trivial. Assume we have shown the following:

Statement 3.7. Let $A$ and $S$ be positive operators inside a masa, which we denote by $\mathcal{A}$, inside a type $I I_{1}$ factor, which we denote by $\mathcal{M}$, so that $A=\sum_{n=1}^{K} \gamma_{n}\left(P_{r_{n}}-\right.$ $\left.P_{r_{n-1}}\right)$ and $S=\sum_{n=1}^{K} \delta_{n}\left(P_{r_{n}}-P_{r_{n-1}}\right)$ for some sequences $0=r_{0}<r_{1}<\cdots<$ $r_{K-1}<r_{K}=1, \gamma_{1} \geq \cdots \geq \gamma_{K}, \delta_{1} \geq \cdots \geq \delta_{K}$, where $K$ is a natural number less than $L$. Then, there is a unitary $U$ so that $E\left(U S U^{*}\right)=A$. 
We will now show that we can extend this to the case when the decompositions have length $L$ as well. To avoid trivialities, we assume that $A \neq S$.

The majorization condition for the operators $A$ and $S$ that we are working with becomes the following: $\tau(A)=\tau(S)$, and for every $k=1, \cdots, L-1$ we have that

$$
\int_{0}^{r_{k}} f(r) d m(r)=\sum_{n=1}^{k} \gamma_{n}\left(r_{n}-r_{n-1}\right) \leq \sum_{n=1}^{k} \delta_{n}\left(r_{n}-r_{n-1}\right)=\int_{0}^{r_{k}} g(r) d m(r) .
$$

In particular, $\gamma_{1} \leq \delta_{1}$. If $\gamma_{n} \leq \delta_{n}$ for every $n=1, \cdots, L$ (and since $A \neq S$, at least one of these expressions must be a strict inequality), then

$$
\tau(A)=\sum_{n=1}^{L} \gamma_{n}\left(r_{n}-r_{n-1}\right)<\sum_{n=1}^{L} \delta_{n}\left(r_{n}-r_{n-1}\right)=\tau(S)
$$

which contradicts the fact that $A \prec S$ (which entails that $\tau(A)=\tau(S)$ ). Thus, there is a natural number $1<l \leq L$ so that

$$
\gamma_{n}<\delta_{n} \text { for } n=1, \cdots, l \text { and } \gamma_{l+1}>\delta_{l+1} \text {. }
$$

Suppose that $\left(\delta_{l}-\gamma_{l}\right)\left(r_{l}-r_{l-1}\right) \leq\left(\gamma_{l+1}-\delta_{l+1}\right)\left(r_{l+1}-r_{l}\right)$ (the other case is handled similarly). Pick $r$ so that $r_{l}<r \leq r_{l+1}$ and such that $\left(\delta_{l}-\gamma_{l}\right)\left(r_{l}-r_{l-1}\right)=$ $\left(\gamma_{l+1}-\delta_{l+1}\right)\left(r-r_{l}\right)$. Let

$A_{1}:=\gamma_{l}\left(P_{r_{l}}-P_{r_{l-1}}\right)+\gamma_{l+1}\left(P_{r}-P_{r_{l}}\right) \quad$ and $\quad S_{1}:=\delta_{l}\left(P_{r_{l}}-P_{r_{l-1}}\right)+\delta_{l+1}\left(P_{r}-P_{r_{l}}\right)$.

Then

$$
\tau\left(S_{1}-A_{1}\right)=\left(\delta_{l}-\gamma_{l}\right)\left(r_{l}-r_{l-1}\right)+\left(\delta_{l+1}-\gamma_{l+1}\right)\left(r-r_{l}\right)=0
$$

Combining this with the fact that $\gamma_{l}<\delta_{l}$ and using Lemma 3.2, we conclude that $A_{1} \prec S_{1}$ inside the $I I_{1}$ factor $P \mathcal{M} P$ where $P$ is the projection $P=P_{r}-P_{r_{l-1}}$. Now, let

$$
A_{2}:=A-A_{1}=\gamma_{l+1}\left(P_{r_{l+1}}-P_{r}\right)+\sum_{n \neq l, l+1} \gamma_{n}\left(P_{r_{n}}-P_{r_{n-1}}\right)
$$

and, similarly,

$$
S_{2}:=S-S_{1}=\delta_{l+1}\left(P_{r_{l+1}}-P_{r}\right)+\sum_{n \neq l, l+1} \delta_{n}\left(P_{r_{n}}-P_{r_{n-1}}\right)
$$

where the operators are considered in $(I-P) \mathcal{M}(I-P)$. We have

(1) $\sum_{n=1}^{k} \gamma_{n}\left(r_{n}-r_{n-1}\right) \leq \sum_{n=1}^{k} \delta_{n}\left(r_{n}-r_{n-1}\right)$ for $k=1, \cdots, l-1$. 
(2) For $k \geq l+1$ (if $k=l+1$, the third term in the first expression below will not show up)

$$
\begin{aligned}
& \sum_{n=1}^{l-1} \gamma_{n}\left(r_{n}-r_{n-1}\right)+\gamma_{l+1}\left(r_{l+1}-r\right)+\sum_{n=l+2}^{k} \gamma_{n}\left(r_{n}-r_{n-1}\right) \\
= & \sum_{n=1}^{k} \gamma_{n}\left(r_{n}-r_{n-1}\right)-\tau\left(A_{1}\right) \\
& <\sum_{n=1}^{k} \delta_{n}\left(r_{n}-r_{n-1}\right)-\tau\left(A_{1}\right) \\
& =\sum_{n=1}^{k} \delta_{n}\left(r_{n}-r_{n-1}\right)-\tau\left(S_{1}\right) \\
& =\sum_{n=1}^{l-1} \delta_{n}\left(r_{n}-r_{n-1}\right)+\delta_{l+1}\left(r_{l+1}-r\right)+\sum_{n=l+2}^{k} \delta_{n}\left(r_{n}-r_{n-1}\right) . \\
\text { (3) } \tau\left(A_{2}\right)= & \tau(A)-\tau\left(A_{1}\right)=\tau(S)-\tau\left(S_{1}\right)=\tau\left(S_{2}\right) .
\end{aligned}
$$

We thus conclude that we also have that $A_{2} \prec S_{2}$.

By Proposition [3.4, there is a unitary $U_{1}$ inside $P \mathcal{M} P$ so that $E\left(U_{1} S_{1} U_{1}^{*}\right)$ $=A_{1}$. Also, the induction hypothesis holds for the operators $A_{2}$ and $M_{2}$ inside $(I-P) \mathcal{M}(I-P)$ since the partition decomposition for $A_{2}$ and $S_{2}$ has length $L-1$. We may therefore find a unitary $U_{2}$ inside so that $E\left(U_{2} S_{2} U_{2}^{*}\right)=A_{2}$. Thus, letting $U=U_{1} \oplus U_{2}$, we have that $E\left(U S U^{*}\right)=A$.

\section{An approximate Schur-Horn theOrem}

Theorem 3.6] allows us to prove an approximate version of the Schur-Horn theorem for general positive operators.

Theorem 4.1. Let $S$ be a positive operator in a $I I_{1}$ factor $\mathcal{M}$ and let $\mathcal{A}$ be a masa in $\mathcal{M}$. Then, the norm closure of $E(\mathcal{U}(S))$ equals $\left\{A \in \mathcal{A}^{+} \mid A \prec S\right\}$.

Proof. Choose $A$ in $\mathcal{A}^{+}$so that $A \prec S$. By scaling, if needed, we assume that $A$ and $S$ are strict contractions. Fix $n>0$ and define the mutually orthogonal projections

$$
P_{k}=E_{A}\left(\left[\frac{k-1}{n}, \frac{k}{n}\right)\right) \quad \text { for } \quad 1 \leq k \leq n .
$$

Next, define $\alpha_{k}=\tau\left(A P_{k}\right)$ for $1 \leq k \leq n$ and consider the operator $B=\sum_{k=1}^{n} \alpha_{k} P_{k}$. Since $\tau(C) I \prec C$ for any positive operator $C$, we have that $B \prec A$, and hence $B \prec S$. We also have that

$$
\|A-B\|=\left\|\sum_{k=1}^{n}\left(A-\alpha_{k}\right) P_{k}\right\| \leq\left\|\sum_{k=1}^{n} \frac{1}{n} P_{k}\right\|=\frac{1}{n} .
$$

Choose numbers $0=t_{0} \leq t_{1} \leq t_{2} \leq \cdots \leq t_{n}=1$ and orthogonal projections $Q_{1}, \cdots, Q_{n}$ in $\left\{S^{\prime} \cap \mathcal{M}\right\}$ such that $Q_{k} \leq E_{S}\left(\left[t_{k-1}, t_{k}\right]\right)$ and $\tau\left(Q_{k}\right)=\tau\left(P_{k}\right)$ for $1 \leq k \leq n$. To see why this possible, proceed as follows: Let $t_{0}=0$ and pick $t_{1}$ such that $\tau\left(E_{S}\left(\left[0, t_{1}\right)\right)\right) \leq \tau\left(P_{1}\right) \leq \tau\left(E_{S}\left(\left[0, t_{1}\right]\right)\right)$. If $S$ has no atom at $t_{1}$, then let $Q_{1}=E_{S}\left(\left[0, t_{1}\right)\right)$. If $S$ has an atom at $t_{1}$, pick a subprojection $R$ of $E_{S}\left(\left\{t_{1}\right\}\right)$ such that $\tau\left(E_{S}\left(\left[0, t_{1}\right)\right)\right)+\tau(R)=\tau\left(P_{1}\right)$ and let $Q_{1}=E_{S}\left(\left[0, t_{1}\right)\right)+R$. Continue this process for $n=2, \cdots$. 
Next, pick positive operators $T_{1}, \cdots, T_{n}$ all with finite spectrum such that for $1 \leq k \leq n$,

$$
T_{k} \prec S Q_{k}, \quad \tau\left(T_{k}\right)=\tau\left(S Q_{k}\right) \quad \text { and } \quad\left\|S Q_{k}-T_{k}\right\| \leq \frac{1}{n} .
$$

This is done in exactly the same way as the choice of the operator $B$ given the operator $A$, in the first part of this proof. Let $T$ be the operator $T=T_{1}+\cdots+T_{n}$. Then, the above conditions imply

Also, for $1 \leq k \leq m$,

$$
T \prec S \quad \text { and } \quad\|S-T\| \leq \frac{1}{n} .
$$

$\tau\left(T\left(Q_{1}+\cdots+Q_{k}\right)\right)=\tau\left(S\left(Q_{1}+\cdots+Q_{k}\right)\right) \geq \tau\left(A\left(P_{1}+\cdots+P_{k}\right)\right)=\tau\left(B\left(P_{1}+\cdots+P_{k}\right)\right)$.

The middle inequality is because $A \prec S$. Hence, by Lemma 3.5, $B \prec T$. Since $B$ and $T$ have finite spectrum, there is a unitary $U$ so that $B=E\left(U T U^{*}\right)$. We calculate that

$\left\|A-E\left(U S U^{*}\right)\right\| \leq\|A-B\|+\left\|B-E\left(U T U^{*}\right)\right\|+\left\|E\left(U T U^{*}-U S U^{*}\right)\right\| \leq \frac{1}{n}+0+\frac{1}{n}$

and see that $A$ can be arbitrarily well approximated by elements in $E(\mathcal{U}(S))$. Since $A$ was arbitrary, we have that the norm closure of $E(\mathcal{U}(S))$ equals $\left\{A \in \mathcal{A}^{+}\right.$। $A \prec S\}$.

\section{Discussion}

The proofs given above can be easily adapted to masas in type $I I I$ factors that admit a faithful normal conditional expectation. Cartan masas by definition satisfy this property, but not all masas do; by a result of Takesaki [12], if every masa in a von Neumann algebra admits a normal conditional expectation, then it is finite. Suppose $\mathcal{A}$ is a masa in a type $I I I$ factor $\mathcal{M}$ admitting a normal conditional expectation $E: \mathcal{M} \rightarrow \mathcal{A}$. Let $A \in \mathcal{A}$ and $S$ be positive operators. For any self-adjoint operator $T$, let $\alpha(T)=\min (\{x \in \sigma(T)\})$. For any unitary $U$ in $\mathcal{M}$, we have that $\left\|E\left(U S U^{*}\right)\right\| \leq\|S\|$ and that $\alpha\left(E\left(U S U^{*}\right)\right) \geq \alpha(S)$. It is now easy to see that a necessary condition for the existence of an element $T \in \mathcal{O}(S)$ such that $E(T)=A$ is that $\|A\| \leq\|S\|$ and $\alpha(A) \geq \alpha(S)$.

The Schur-Horn problem in type $I I I$ factors is more tractable that in the type $I I_{1}$ case. Standard arguments allow us to prove the following lemma.

Lemma 5.1. Let $S=\sum_{n=1}^{N} \mu_{n} F_{n}$ be a positive contraction with finite spectrum in a type III factor $\mathcal{M}$ with $\|S\|=1$ and $\alpha(S)=0$. Then, $\mathcal{O}(S)$ contains a non-trivial projection (and thus every projection).

With this in hand, it is easy to see that if $A \in \mathcal{A}$ and $S \in \mathcal{M}$ are positive elements with finite spectrum so that $\|A\| \leq\|S\|$ and $\alpha(A) \geq \alpha(S)$, then we can solve the Schur-Horn problem for $A$ and $S$. There is further a simple condition that allows us to determine when we can find a unitary so that $E\left(U S U^{*}\right)=A$.

Suppose 0 is the point spectrum of $A$ so that there is a projection $P$ in $\mathcal{A}$ so that $P A P=0$. Suppose we write $A=E(T)$ for some positive operator $T$; then $E(P T P)=0$ and hence $P T P=0$. Thus, 0 must be in the point spectrum of $T$. If $A=E\left(U S U^{*}\right)$, we get that 0 must be in the point spectrum of $U S U^{*}$ and hence in the point spectrum of $S$. Similarly, if 1 is in the point spectrum of a positive contraction $A$ and $A=E\left(U S U^{*}\right)$ for some positive contraction $S$ and a unitary $U$, 
then 1 must be in the point spectrum of $S$ as well. These necessary conditions are also sufficient.

Theorem 5.2. Let $\mathcal{A}$ be a masa inside a type III factor $\mathcal{M}$ admitting a faithful normal conditional expectation $E$, let $A$ and $S$ be positive operators with finite spectrum in $\mathcal{A}$ and $\mathcal{M}$ respectively and let $E$ be the normal conditional expectation onto $\mathcal{A}$. Assume further that $\alpha(A) \geq \alpha(S)$ and $\|A\| \leq\|S\|$. Then:

(1) There is an element $T \in \mathcal{O}(S)$ such that $E(T)=A$.

(2) Assume additionally that if either 0 and $\|S\|$ are in the point spectrum of $A$, then they are in the point spectrum of $S$ as well. Then, there is a unitary $U$ such that $E\left(U S U^{*}\right)=A$.

We omit the details, as they are a straightforward adaptation of the proof of Theorem 3.6 .

In general, we could ask:

Question 5.3. Let $A$ and $S$ be positive operators in $\mathcal{A}$ and $\mathcal{M}$ respectively, where $\mathcal{A}$ is a masa inside a type $I I I$ factor admitting a normal conditional expectation and so that $\|A\| \leq\|S\|$ and $\alpha(A) \geq \alpha(S)$. Then, is there an element $T$ in $\mathcal{O}(S)$ so that $E(T)=A$ ?

Lyapunov's theorem [10, which states that the range of any non-atomic vector valued measure taking values in $\mathbb{C}^{n}$ is compact and convex, was reformulated in operator algebraic language by Lindenstrauss [9] to say the following: Let $\Phi$ be a weak* continuous linear map from a non-atomic abelian von Neumann algebra into $\mathbb{C}^{n}$. Then, for any positive contraction $A$, there is a projection $P$ such that $\Phi(A)=\Phi(P)$. Akemann and Anderson, in their superb monograph [2, called any theorem concerning linear maps $\Phi: \mathcal{X} \rightarrow \mathcal{Y}$, where $\mathcal{X}$ and $\mathcal{Y}$ are subsets of linear spaces. This assures us that $\operatorname{Ran}(\Phi)=\operatorname{Ran}(\Phi \mid \partial(\mathcal{X}))$ a Lyapunov type theorem. Clearly, Kadison's carpenter problem is a Lyapunov type problem. Anderson and Akemann proved a variety of Lyapunov theorems and showed, quite surprisingly, that Lyapunov theorems are substantially more tractable when the maps considered are singular. The one of most interest to us is

Theorem 5.4 (Akemann and Anderson). Let $\mathcal{A}$ be a masa in an type $I_{1}$ factor $\mathcal{M}$. Let $F$ be a singular conditional expectation from $\mathcal{M}$ to $\mathcal{A}$. Then, for any positive contraction $A$ in $\mathcal{A}$, there is a projection $P$ in $\mathcal{M}$ so that $F(P)=A$.

There are plenty of singular conditional expectations onto masas in $I I_{1}$ factors [1, though none of them are trace preserving. The corresponding Schur-Horn problem cannot be any other than

Question 5.5. Let $\mathcal{A}$ be a masa in an type $I I_{1}$ factor $\mathcal{M}$. Let $F$ be a singular conditional expectation from $\mathcal{M}$ to $\mathcal{A}$. Suppose $A \in \mathcal{A}$ and $S \in \mathcal{M}$ are positive contractions that are not multiples of the identity such that $\|A\| \leq\|S\|$ and $\alpha(A) \geq$ $\alpha(S)$. Then, is there an element $T \in \mathcal{O}(S)$ such that $F(T)=A$ ?

Finally, an answer to the following related question, which we are unable to solve, should help in solving the Schur-Horn and carpenter problems in type $I I_{1}$ factors.

Question 5.6. Let $A$ be a positive operator in a masa $\mathcal{A}$ inside a $I I_{1}$ factor $\mathcal{M}$. Then, does the norm closure of $\mathfrak{L}(A)=\{S \in \mathcal{M} \mid \exists T \in \mathcal{O}(S)$ so that $E(T)=A\}$ equal $\{S \in \mathcal{M} \mid A \prec S\}$ ? 


\section{ACKNOWLEDGEMENTS}

The authors would like to thank Sabanci University for a research grant that supported the visit of the first author to Sabanci University, Istanbul, in September 2011, when part of this work was done. The second author would also like to thank Matt Daws for pointing out Mathoverflow, a result of Takesaki that is mentioned in the last section.

\section{REFERENCES}

[1] Charles Akemann and David Sherman, Conditional expectations onto maximal abelian *-subalgebras. J. Operator Theory 68 (2012), no. 2, 597-607. MR2995737

[2] Charles A. Akemann and Joel Anderson, Lyapunov theorems for operator algebras, Mem. Amer. Math. Soc. 94 (1991), no. 458, iv+88. MR1086563(92e:46113)

[3] Martin Argerami and Pedro Massey, A Schur-Horn theorem in $\mathrm{II}_{1}$ factors, Indiana Univ. Math. J. 56(5) (2007), 2051-2059. MR2359722

[4] William Arveson and Richard V. Kadison, Diagonals of self-adjoint operators, Operator theory, operator algebras, and applications, Contemp. Math., vol. 414, Amer. Math. Soc., Providence, RI, 2006, pp. 247-263, DOI 10.1090/conm/414/07814. MR2277215 (2007k:46116)

[5] Ken Dykema, Junsheng Fang, Don Hadwin, and Roger Smith, The Carpenter and SchurHorn problems for masas in finite factors. arXiv: 1110.6500

[6] Fumio Hiai, Majorization and stochastic maps in von Neumann algebras, J. Math. Anal. Appl. 127 (1987), no. 1, 18-48, DOI 10.1016/0022-247X(87)90138-7. MR.904208 (88k:46076)

[7] Richard V. Kadison, The Pythagorean theorem. I. The finite case, Proc. Natl. Acad. Sci. USA 99 (2002), no. 7, 4178-4184 (electronic), DOI 10.1073/pnas.032677199. MR1895747 (2003e:46108a)

[8] Thierry Fack and Hideki Kosaki, Generalized s-numbers of $\tau$-measurable operators, Pacific J. Math. 123 (1986), no. 2, 269-300. MR840845 (87h:46122)

[9] Joram Lindenstrauss, A short proof of Liapounoff's convexity theorem, J. Math. Mech. 15 (1966), 971-972. MR0207941 (34 \#7754)

[10] Aleksei Lyapunov, On completely additive vector functions, Izv. Akad. Nauk SSSR 4 (1940), 465-478. MR0004080(2,315e)

[11] Sorin Popa, On a problem of $R$. V. Kadison on maximal abelian *-subalgebras in factors, Invent. Math. 65 (1981/82), no. 2, 269-281, DOI 10.1007/BF01389015. MR641131 (83g:46056)

[12] Masamichi Takesaki, Conditional expectations in von Neumann algebras, J. Functional Analysis 9 (1972), 306-321. MR0303307 (46 \#2445)

Indian Statistical Institute, R V College Post, Bangalore, India 560059

E-mail address: bhat@isibang.ac.in

Department of Mathematics, Istanbul Bilgi University, Dolapdere, Istanbul, Turkey 34440

Current address: Department of Mathematics, Mimar Sinan Fine Arts University, Bomonti, Istanbul, Turkey 34400 